\title{
Evolving contraceptive options in indigenous communities in Panama
}

Alyssa Jacob, Rachel Wallice, Megan Lewis \& Ben La Brot - Floating Doctors, Panama

\section{Background}

Panama is a Central American country with a total population of approximately 3.9 million. Panama's indigenous population accounts for $12.3 \%$. The Ngäbe-Buglé is the largest indigenous group, representing $68.3 \%$ of the total indigenous population. A large proportion of the Ngäbe-Buglé live in isolated, rural communities with limited sanitation and education facilities and poor access to local health services.

Prevalence of contraception use and numbers of births attended by a qualified professional are significantly lower in indigenous groups than nationally. Birth-rates and maternal and infant mortality rates in the Ngäbe-Buglé are among the highest in the country (table 1).

A non-governmental organization (NGO), Floating Doctors, has been providing free primary healthcare to Ngäbe-Buglé communities in remote areas of the Bocas del Toro region since 2011. A study undertaken by the NGO in 2014 identified family planning as an unmet need; $55 \%$ of women of childbearing age who were not using contraception did not want further children. Floating Doctors distribute condoms and in 2013 began to provide the contraceptive injection.

\section{Aims}

To assess the level and trends of contraceptive injection use and to identify associated challenges.

\section{Methods}

An observational study of depot medroxyprogesteroneacetate (DMPA) use in women attending the NGO clinics between January 2013 and June 2015 was carried out. In addition, a focus group was conducted using a semi-structured interview approach to explore the views, experiences and beliefs of the Ngäbe-Buglé women regarding the contraceptive injection.

Figure 1: reasons for missed doses

Table 1: birth-rates and maternal and infant mortality rates

\begin{tabular}{l|c|c|} 
& National & $\begin{array}{c}\text { Ngäbe- } \\
\text { Buglé }\end{array}$ \\
\hline $\begin{array}{l}\text { Birth-rate per } 1,000 \\
\text { (2009) }\end{array}$ & 19.8 & 33.4 \\
\hline $\begin{array}{l}\text { Maternal mortality rate per } \\
\begin{array}{l}100,000 \text { (2010) } \\
\text { Infant mortality rate per 1,000 } \\
(2010)\end{array}\end{array}$ & 24.9 & 90 \\
\hline
\end{tabular}

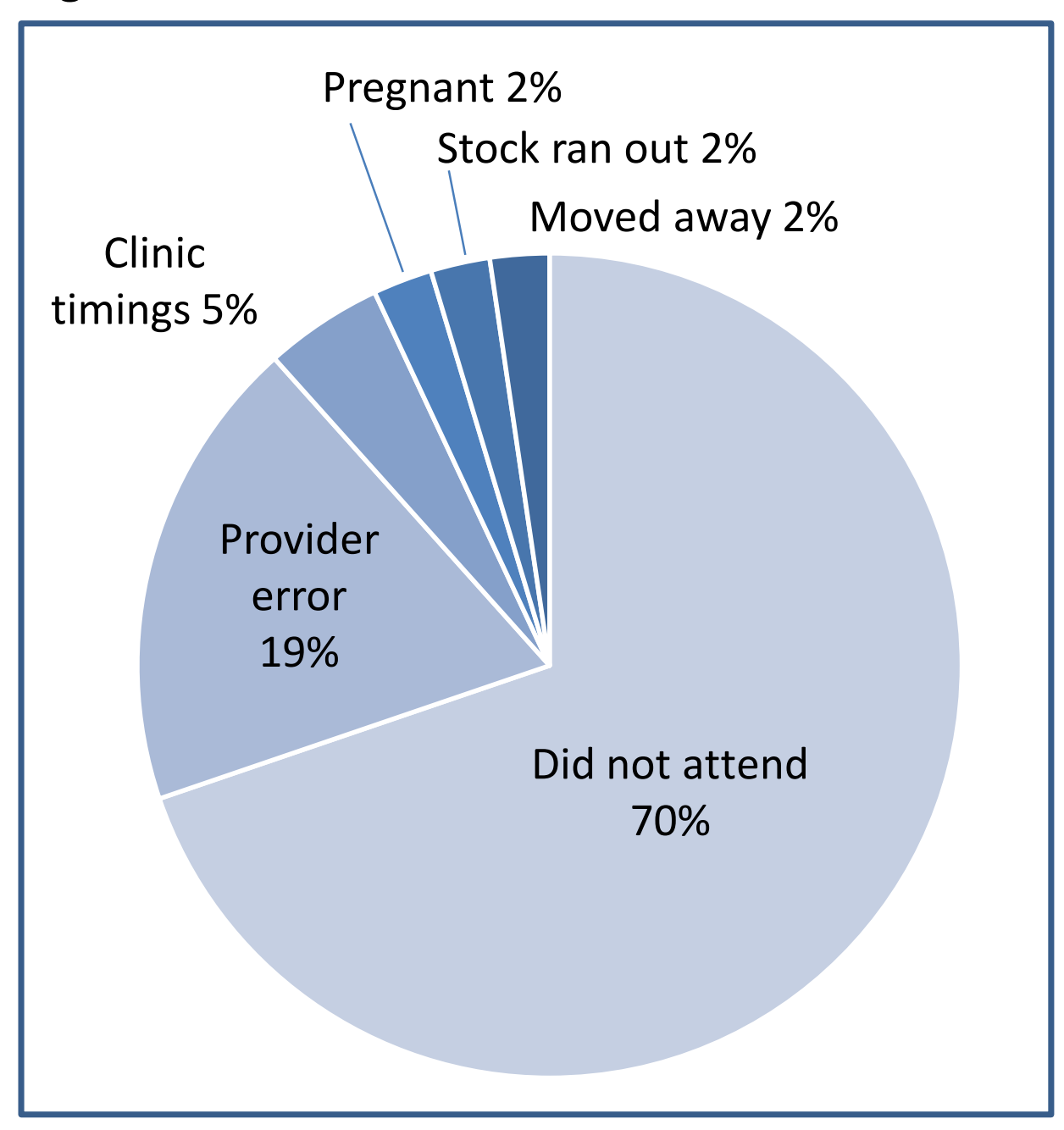

Results

43 women from 16 communities have used DMPA from the NGO; 46.9\% had started in the last 6 months of the study period. The most common reason for commencing is family completion. Average age at commencing is 27.6 years (range 12-46) and number of children is 4 (range $0-14$ ). Since starting, $13.3 \%$ have discontinued use and $25.9 \%$ have missed their most recent dose. Missed doses are commonly due to clinic non-attendance (figure 1). Influence from spouses and misconceptions regarding side-effects are key factors in discontinuation.

\section{Discussion}

Ngäbe-Buglé communities are experiencing unsustainable population growth. Family planning is recognised as an unmet need, however contraceptive options available to these communities remain limited. Uptake of DMPA from the NGO varies considerably between different communities but has recently surged. However, major challenges regarding long-term use and compliance remain. Our ongoing work aims to broaden contraceptive options for these people and includes implementation of a pilot study introducing the contraceptive implant. 